\title{
Functional and population genetic features of copy number variations in two dairy cattle populations
}

\author{
Young-Lim Lee ${ }^{1 *} \mathbb{D}$, Mirte Bosse ${ }^{1}$, Erik Mullaart ${ }^{2}$, Martien A. M. Groenen ${ }^{1}$, Roel F. Veerkamp ${ }^{1}$ and \\ Aniek C. Bouwman ${ }^{1}$
}

\begin{abstract}
Background: Copy Number Variations (CNVs) are gain or loss of DNA segments that are known to play a role in shaping a wide range of phenotypes. In this study, we used two dairy cattle populations, Holstein Friesian and Jersey, to discover CNVs using the Illumina BovineHD Genotyping BeadChip aligned to the ARS-UCD1.2 assembly. The discovered CNVs were investigated for their functional impact and their population genetics features.

Results: We discovered 14,272 autosomal CNVs, which were aggregated into 1755 CNV regions (CNVR) from 451 animals. These CNVRs together cover $2.8 \%$ of the bovine autosomes. The assessment of the functional impact of CNVRs showed that rare CNVRs (MAF $<0.01$ ) are more likely to overlap with genes, than common CNVRs (MAF $\geq$ 0.05). The Population differentiation index (Fst) based on CNVRs revealed multiple highly diverged CNVRs between the two breeds. Some of these CNVRs overlapped with candidate genes such as MGAM and ADAMTS17 genes, which are related to starch digestion and body size, respectively. Lastly, linkage disequilibrium (LD) between CNVRs and BovineHD BeadChip SNPs was generally low, close to 0, although common deletions (MAF $\geq 0.05$ ) showed slightly higher $L D\left(r^{2}=\sim 0.1\right.$ at $10 \mathrm{~kb}$ distance) than the rest. Nevertheless, this LD is still lower than SNP-SNP LD $\left(r^{2}=\sim 0.5\right.$ at $10 \mathrm{~kb}$ distance $)$.

Conclusions: Our analyses showed that CNVRs detected using BovineHD BeadChip arrays are likely to be functional. This finding indicates that CNVs can potentially disrupt the function of genes and thus might alter phenotypes. Also, the population differentiation index revealed two candidate genes, MGAM and ADAMTS17, which hint at adaptive evolution between the two populations. Lastly, low CNVR-SNP LD implies that genetic variation from CNVs might not be fully captured in routine animal genetic evaluation, which relies solely on SNP markers.
\end{abstract}

Keywords: Copy number variations, Bos taurus, Linkage disequilibrium, Population genetics

\section{Background}

Genetic variations exist in various forms in genomes. Although single nucleotide polymorphisms (SNPs) have been the choice of variants in numerous studies, there is a growing body of evidence that copy number variations (CNVs) can have functional impact. Copy number variations are DNA segments of $1 \mathrm{~kb}$ or larger, and are present in varying copy numbers, compared to a reference genome [1]. Since the initial discovery of large sub-microscopic CNVs (some hundred

\footnotetext{
* Correspondence: younglim.lee@wur.nl

${ }^{1}$ Wageningen University \& Research, Animal Breeding and Genomics, P.O.

Box 338, Wageningen, $\mathrm{AH} 6700$, the Netherlands

Full list of author information is available at the end of the article
}

kb) $[2,3]$, rapid developments in detection platforms and algorithms have advanced knowledge about CNVs, mainly in humans $[4,5]$.

In the early phase of their discovery, CNVs were expected to resolve the missing heritability (significant SNPs identified from genome-wide association studies (GWAS) together account small part of the heritability) $[6,7]$. It was because, as in terms of base pairs, they cover a larger proportion of the genome, compared to SNPs. With the accumulation of data and analyses, the occurrence of CNVs in the genome was shown to be biased outside of functional elements [5]. Nevertheless, numerous studies have shown that $\mathrm{CNVs}$ play a role in determining a wide range of

(c) The Author(s). 2020 Open Access This article is distributed under the terms of the Creative Commons Attribution 4.0 International License (http://creativecommons.org/licenses/by/4.0/), which permits unrestricted use, distribution, and reproduction in any medium, provided you give appropriate credit to the original author(s) and the source, provide a link to the Creative Commons license, and indicate if changes were made. The Creative Commons Public Domain Dedication waiver (http://creativecommons.org/publicdomain/zero/1.0/) applies to the data made available in this article, unless otherwise stated. 
human health conditions, from obesity to neurodevelopmental diseases [8-11]. For instance, high copy numbers of the CCL3L1 and CYP2D6 genes confer reduced susceptibility to infection with HIV and the development of AIDS [12]. Also, the role of CNVs in adaptive evolution is further exemplified by mean copy numbers of the $A M Y 1$ gene (which codes for amylase alpha1, an essential enzyme for starch digestion). The mean copy number of $A M Y 1$ gene was shown to differ in human populations depending on dietary starch composition [13]. These findings demonstrate that CNVs may contribute to adaptive potential, and thus contain information about population history.

Studies in livestock species also highlighted the role of CNVs in shaping various phenotypes. For example, several genes affected by CNVs determine coat colours of specific breeds. Duplications of the KIT gene in pigs are related to white coat, which is only shown in domestic pigs [14, 15]. In cattle, serial translocation of the KIT gene was related to a colour-sidedness phenotype [16]. Moreover, CNVs were shown to be associated with quantitative traits that are economically important in livestock breeding, in various cattle populations [17-19]. One study investigated whether trait associated $\mathrm{CNVs}$ are in linkage disequilibrium (LD) with, and thus are tagged by, SNP markers, and revealed that $25 \%$ of CNVs were not in LD with SNP markers [17]. However, this study was based on Illumina BovineSNP50 array data, in which SNP density and CNV resolution were low.

Holstein Friesian (HOL) and Jersey (JER) are the two main commercial dairy cattle breeds that have been bred under different breeding schemes. Although there have been studies investigating the link between CNVs and individual production traits [17-21], in-depth assessment of functional impacts of CNVs in cattle genomes has been limited. Also, whether CNVs that have an impact on phenotypes are captured in genomic evaluation, in other words, whether CNVs are in sufficient LD with SNPs, is largely unexplored. Furthermore, CNVs have been shown to be useful in disentangling population history and provide valuable insights in understanding how populations have evolved over time [22-25]. However, population genetics analyses exploring CNVs, with their main focus on HOL and JER, have been sparse.

Here, we aimed at discovering CNVs in bovine genomes based on genome assembly ARS-UCD1.2 [26] using high density SNP array data, in two dairy cattle populations. Subsequently, we performed in-depth analyses on the functional impact of CNVs and further explored the population genetic features of $\mathrm{CNVs}$ by analysing population differentiation index (Fst) and LD.

\section{Results}

\section{CNV discovery in the genome build ARS-UCD1.2}

The data consisted of Illumina BovineHD BeadChip (Illumina, San Diego, CA, USA) genotypes from two distinct dairy breeds (Holstein Friesian - HOL $(n=331)$, Jersey - JER $(n=115))$ and their crossbreds $(n=29)$. A previous study using PennCNV on BovineHD data, of which $47 \mathrm{HOL}$ animals overlapped with our study, showed high rate of CNV confirmation based on qPCR validation $(91.7 \%$ for CNVs found in multiple animals, $40 \%$ for singleton CNVs) [24]. Therefore, we chose to perform CNV detection on bovine autosomes using the PennCNV software [27]. The Bovine HD SNPs were aligned to genome assembly ARS-UCD1.2.

We discovered 14,272 CNV calls from 451 individuals that passed the quality control criteria (31.6 calls/individual). Deletion calls were 1.8 times more frequent but $40 \%$ shorter $(n=9171$, mean length $=44.2 \mathrm{~kb})$ than $\mathrm{du}$ plication calls $(n=5101$, mean length $=74.6 \mathrm{~kb}$; Additional file 2: Table S1 and Additional file 1: Figure S1). The mean probe density (number of supporting SNPs per Mb CNV) was $403 \mathrm{SNPs} / \mathrm{Mb}$. The 14,272 CNV calls were aggregated into $1755 \mathrm{CNV}$ regions (CNVRs), based on at least $1 \mathrm{bp}$ overlap, following Redon et al. [28]. These CNVRs cover $2.8 \%$ of the autosomal genome sequence (69.6/2489.4 Mb; Fig. 1; A full list of CNVR is in Additional file 2: Table S2.). These CNVRs consist of 1125 deletion CNVRs (mean length $=29.2 \mathrm{~kb}$ ), $513 \mathrm{du}$ plication CNVRs (mean length $=36.8 \mathrm{~kb}$ ), and $117 \mathrm{com}$ plex CNVRs (mean length $=152.7 \mathrm{~kb}$ ). The distribution of CNVR length is exponential, where the majority CNVRs are short to medium length $(<100 \mathrm{~kb}, 93 \%)$, while only a few observations are made for long CNVRs (>100 kb, 7\%). The CNVRs are non-randomly distributed over the chromosomes: chromosome-wide CNVR coverage varies from $0.6 \%$ on BTA24 to $4.9 \%$ on BTA12 (Additional file 2: Table S3). BTA12 is most densely covered with CNVR in terms of bp (4.2 Mb), and especially enriched for complex type CNVRs (2.2 Mb). Allele frequency of CNVRs ranges between 0.001 and 0.21 .

Since most cattle CNV studies used genome assembly UMD3.1, we also repeated the CNV detection procedures, using UMD3.1. Subsequently, we used these calls to assess our $\mathrm{CNV}$ discovery results with other cattle CNV papers. From the 447 individuals that passed the QC criteria, 24, $264 \mathrm{CNVs}$ were called (54.3 calls/individual) and the mean probe density was $326 \mathrm{SNPs} / \mathrm{Mb}$. These CNVs were aggregated into 1866 CNVRs (1130 deletions, 593 duplications, and 143 complex CNVRs). The mean length of deletion, duplication, and complex CNVRs is 29, 36, and $193 \mathrm{~kb}$, respectively (Additional file 2: Table S1). These CNVRs together cover $82 \mathrm{Mb}(3.3 \%)$ of bovine autosomes. The chromosome-wide coverage varies between $1 \%$ on BTA24 and $10 \%$ on BTA12 (Additional file 2: Table S4 and Additional file 1: Figure S2). Compared to other cattle CNV studies conducted using the same SNP array and the genome assembly UMD3.1 [22, 24, 29-32], our CNV discovery results are in a similar range (Additional file 2: Table S5). 


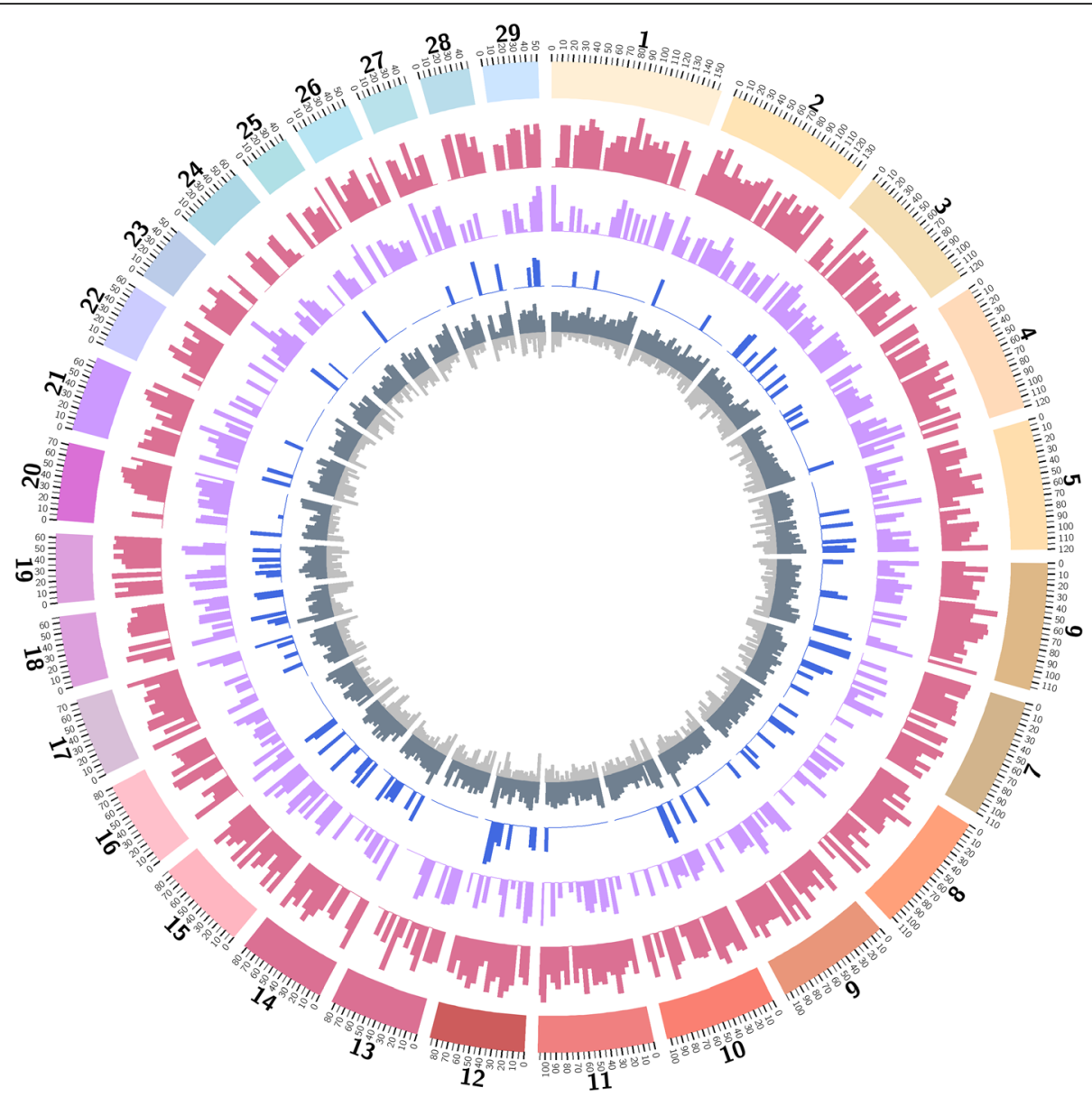

Fig. 1 Circular map of autosomal copy number variant regions and their population genetics features. From the outside to the inside of the external circle: chromosome name; genomic location (in Mb); histogram representing density of deletion CNVRs in $5 \mathrm{Mb}$ bin (pink); histogram representing density of duplication CNVRs in $5 \mathrm{Mb}$ bin (purple); histogram representing density of complex CNVRs in $5 \mathrm{Mb}$ bin (blue); number of BovineHD BeadChip array SNPs in $5 \mathrm{Mb}$ bin (dark grey); histogram representing density of segmental duplications in $5 \mathrm{Mb}$ bin (light grey)

When we compared to our CNVs discovered based on UMD3.1 and ARS-UCD1.2, we observed several differences. Firstly, the number of CNVs called per individual based on ARS-UCD1.2 is $42 \%$ lower than what was obtained using UMD3.1. Also, the mean probe density increased from $326 \mathrm{SNPs} / \mathrm{Mb}$ in UMD3.1 to 404 SNPs/ $\mathrm{Mb}$ in ARS-UCD1.2, indicating that with ARS-UCD1.2, CNVs are supported by more SNPs. Lastly, the mean length of complex CNVRs decreased by $40 \mathrm{~kb}$, from 193 $\mathrm{kb}$ in UMD3.1 to $152.7 \mathrm{~kb}$ in ARS-UCD1.2. We further inspected BTA12:70-77 MB region where a large change between UMD3.1 and ARS-UCD1.2 was observed. This region was reported to have a large number deletion and duplication calls by other cattle CNV studies based on UMD3.1, regardless of the studied breeds [24, 29-33]. In our CNV discovery, we identified 7 CNVRs (total length of $\sim 6.2 \mathrm{Mb}$ ) in this region based on UMD3.1, whereas ARS-UCD1.2 based results revealed 9 CNVRs that covered $\sim 1 \mathrm{Mb}$. We compared the positions of BovineHD SNPs in UMD3.1 and ARS-UCD1.2 to see whether the changes in genome assemblies caused this discrepancy. The results showed that $43 \%$ of the SNPs located in BTA12:70-77 Mb based on UMD3.1 were either moved to unmapped contigs or reference and alternative SNPs were undefined. The genome-wide ratio of SNPs that were moved to different chromosomes or contigs was much lower $(2.3 \%)$ than $43 \%$. This indeed indicates that the two genome assemblies differ in this regions, and thus led to different $\mathrm{CNV}$ discovery results.

\section{Functional impact of CNVRs}

The expression of genes can be altered by CNVs. Deletions and duplications of a part of and/or complete gene can disrupt the gene expression and can potentially lead to changes in various phenotypes [34]. Therefore, identification CNVRs that coincide with genes can be a primary step to assess their functional impact. To achieve this, we explored CNVRs found based on ARS-UCD1.2 further. The overlap of CNVRs with Ensembl annotated genes were analysed, and among the 1755 CNVRs, 912 
(52\%) are genic and $843(48 \%)$ are intergenic. Genic CNVRs overlap with 1739 genes out of 27,570 Ensembl annotated genes $(6.3 \%)$ and 2936 out of 43,949 gene transcripts (6.7\%). Among the 1739 genes that overlap with CNVRs, 957 (55\%) are completely within the CNVRs and the rest (45\%) are partially affected (genic features were inside the CNVRs). The following functional impact categories were assigned to each CNVR depending on types of overlap between CNVRs and genes (numbers in the brackets indicate number of CNVRs and genes respectively for each category; see materials and methods for detailed explanation for the classification): 1) intergenic (843 CNVRs; 0 genes), 2) intronic (214 CNVRs; 234 genes), 3) whole gene (253 CNVRs; 957 genes), 4) stop codon (147 CNVRs; 203 genes), 5) promoter regions (124 CNVRs; 187 genes), and 6) exonic (174 CNVRs; 165 genes). Then, these functional categories were intersected with other features of CNVRs such as types (deletion, duplication, complex), MAF (common, intermediate, and rare; see methods for detailed explanation), and the populations (HOL and JER; Fig. 2). The functional consequences of
CNVRs differ depending on the type of CNVRs: Complex CNVRs were skewed towards genic regions $(68 \%$ are genic), whereas deletions and duplication CNVRs were biased away from genic regions (51-52\% are genic), and the difference is significant (chi-square test $P<10^{-13}$ ). Also, we observed that MAF have impact on different types of overlap between genes and CNVRs. Rare CNVRs tend to be genic more often (60\%), whereas common CNVRs have less overlap compared to it (48\%; chi-square test $P<0.002)$. However, when seen it separately for deletion CNVRs and duplication CNVRs, we saw a different pattern. Common deletion CNVRs are more often intergenic (61\%), yet the common duplication CNVRs are often genic (68\%). When CNVRs between HOL and JER are compared, common JER CNVRs are more often genic (51\%), than common HOL CNVRs (44\%). Subsequently, we performed permutation tests on overlaps between CNVRs and autosomal genes, to test whether the overlap is significantly higher than expected under a neutral scenario. The results show that CNVRs overlap with autosomal genes more often than what is expected from

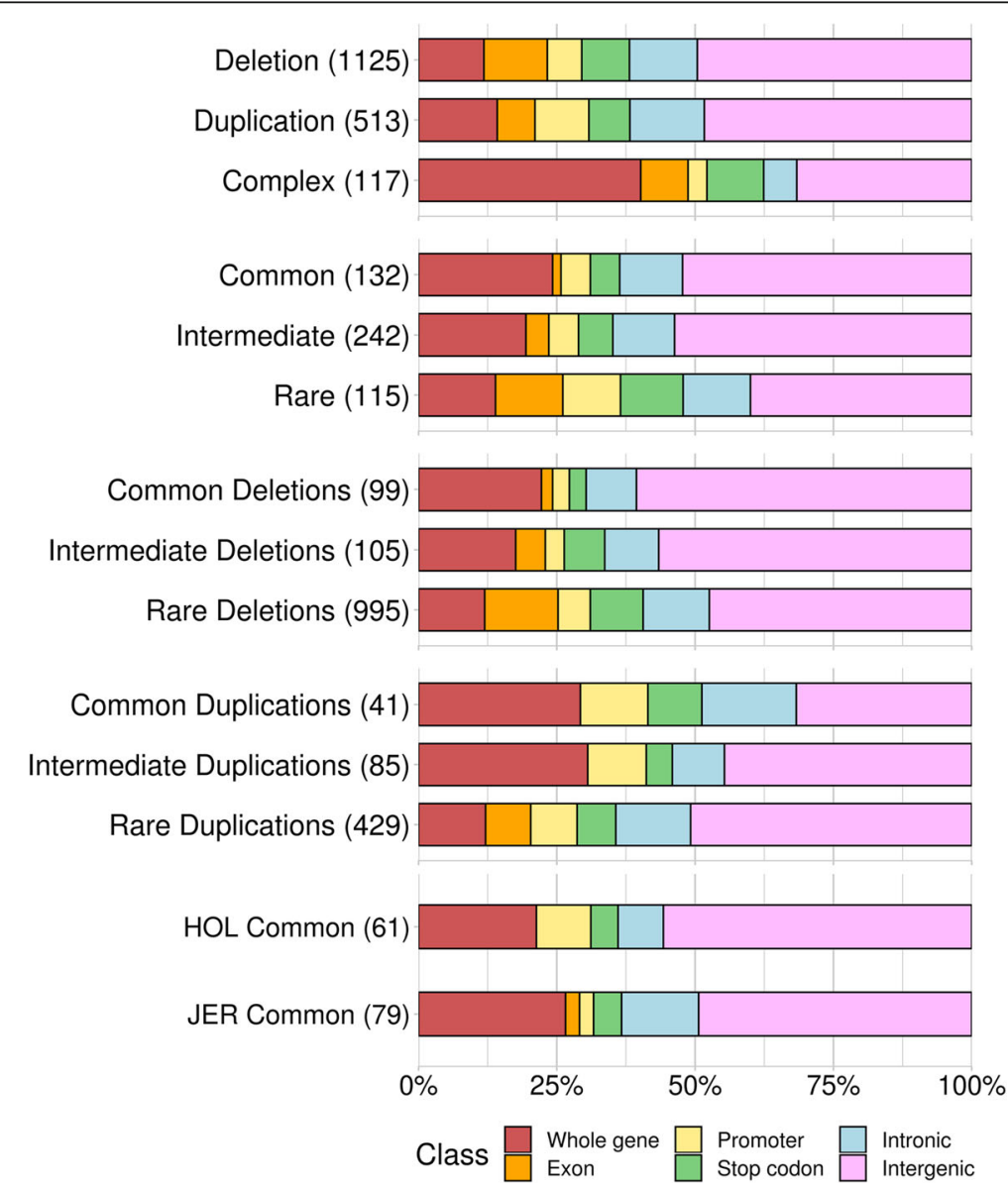

Fig. 2 Functional impact of CNVRs by type, frequency, and population. Functional impact of CNVRs were investigated by type, frequency, and population. CNVRs were categorized into different types (deletion, duplication, and complex) and frequency (common: $0.05 \leq \mathrm{MAF}$ in any population, intermediate: $0.01 \leq \mathrm{MAF}<0.05$, rare: MAF $<0.01$ in all populations). The numbers in the brackets indicate the number of CNVRs in each category 
permutation tests with random genomic regions $(P<$ 0.001 ). Nextly, gene ontology analyses were performed to understand the functions of the genes that overlap with CNVRs. Genes overlapping deletions, duplications, and complex CNVRs were tested for GO enrichment as separate classes (Table 1). Among the findings, genes overlapping with the complex CNVRs $(n=407)$ show a pronounced enrichment in response to stimulus (GO: 0050896; $\mathrm{FDR}=1.8 \times 10^{-6}$ ), immune response (GO: 0006955; FDR $\left.=1.9 \times 10^{-3}\right)$, and detection of stimulus involved in sensory perception (GO:0050906; FDR $=1.1 \mathrm{X}$ $10^{-2}$ ). These findings are similar to the findings from earlier cattle CNV studies [30, 33].

\section{Population genetics of CNVRs}

Population genetics analyses provide a framework to understand genetic variation seen in specific (cattle) populations. Understanding general properties of genetic variants is important, but further characterization of specific variants of interest can bring insights in recent adaptation and genome biology [35]. Although SNPs have been extensively used in characterizing various cattle populations [36], we explored the population genetic properties of CNVRs.

We focused our analyses on HOL $(n=315)$ and JER $(n=107)$ animals, derived from distinct origins and with a different breed formation history [37]. First, we coded the genotypes of our bi-allelic CNVRs $(n=1154$ for HOL; $n=700$ for JER) as "+/+", "+/-", and "-/-". The CNVR allele frequency was classified as rare $(\mathrm{MAF}<$
$0.01)$, intermediate $(0.01 \leq \mathrm{MAF}<0.05)$ and common $(0.05 \leq \mathrm{MAF})$. In HOL, the allele frequency ranged from 0.002 to 0.29 , and 5,13 , and $82 \%$ of the 1154 CNVRs were categorized as common, intermediate, and rare CNVRs, respectively. For the JER population, allele frequency ranged from 0.005 to 0.37 , and 11,20 , and $69 \%$ of the $700 \mathrm{CNVRs}$ were categorized as common, intermediate, and rare CNVRs, respectively.

We constructed site frequency spectra of CNVRs for HOL and JER separately (Fig. 3). For both populations, we observed that deletions and duplications have slightly different spectra, where deletions were more skewed towards rare $\mathrm{CNVs}$, whereas duplications were observed relatively more frequent than deletions in each MAF class. We further explored the allele frequencies by applying Wright's fixation index (Fst) [38] to characterize population structure [39] and detect loci that underwent selection [40], as done in Yali Xue et al. [41]. Given that HOL and JER have distinctive origins and breed formation history [37], we hypothesized that Fst on their CNVRs can reveal regions that underwent recent population differentiation. The Fst distribution followed an exponential decay pattern, as expected, underlining that majority of CNVRs have values close to 0 , whereas only a few outliers $(\sim 3 \%)$ that are potentially under positive selection reached high Fst values (Additional file 2: Figure S3). We identified 32 highly diverged CNVRs (Fst $>$ mean +3 S.D.) of which 15 are genic and 17 are intergenic (Fig. 4 and Additional file 2: Table S6). Among the 17 intergenic CNVRs with high population

Table 1 Go enrichment results for different types of CNVR

\begin{tabular}{|c|c|c|c|c|c|c|}
\hline Type of CNVRs & GO Term & Size & Count & $\begin{array}{l}\text { Expected } \\
\text { count }\end{array}$ & $\begin{array}{l}\text { Enrichment } \\
\text { value }\end{array}$ & $\begin{array}{l}P \text {-value } \\
\text { (FDR corrected) }\end{array}$ \\
\hline DEL & Chemical synaptic transmission & 278 & 22 & 8.3 & 2.65 & 0.126 \\
\hline DEL & Anterograde trans-synaptic signalling & 278 & 22 & 8.3 & 2.65 & 0.063 \\
\hline DEL & Trans-synaptic signalling & 279 & 22 & 8.33 & 2.64 & 0.044 \\
\hline DEL & Synaptic signalling & 279 & 22 & 8.33 & 2.64 & 0.033 \\
\hline DUP & Positive regulation of adaptive immune response & 32 & 6 & 0.44 & 13.76 & 0.019 \\
\hline DUP & Positive regulation of immune response & 57 & 7 & 0.78 & 9.01 & 0.021 \\
\hline DUP & Positive regulation of response to stimulus & 75 & 7 & 1.02 & 6.85 & 0.053 \\
\hline DUP & Adaptive immune response & 108 & 9 & 1.47 & 6.11 & 0.018 \\
\hline DUP & Immune effector process & 104 & 8 & 1.42 & 5.64 & 0.049 \\
\hline COMP & Response to stimulus & 1718 & 45 & 16.63 & 2.71 & 0.000 \\
\hline COMP & Immune response & 298 & 14 & 2.88 & 4.85 & 0.002 \\
\hline COMP & Detection of stimulus involved in sensory perception & 477 & 16 & 4.62 & 3.47 & 0.011 \\
\hline COMP & B cell activation & 17 & 4 & 0.16 & 24.31 & 0.013 \\
\hline COMP & Detection of chemical stimulus involved in sensory perception & 477 & 16 & 4.62 & 3.47 & 0.014 \\
\hline COMP & Detection of stimulus & 501 & 16 & 4.85 & 3.3 & 0.015 \\
\hline COMP & Immune system process & 322 & 12 & 3.12 & 3.85 & 0.025 \\
\hline COMP & B cell receptor signalling pathway & 23 & 4 & 0.22 & 17.97 & 0.027 \\
\hline
\end{tabular}



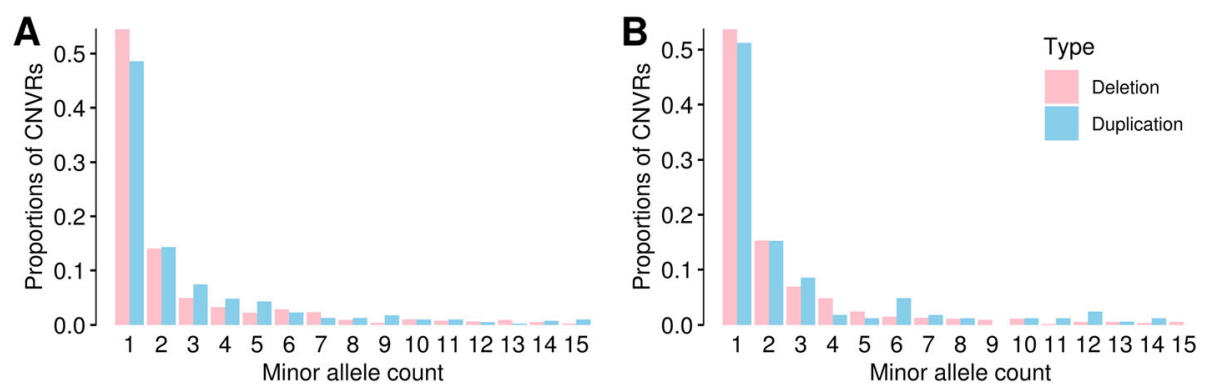

Fig. 3 Site frequency spectrum of CNVRs. Site frequency spectra of CNVRs in HOL (a) and JER (b) population. Deletion CNVRs (pink) and duplication CNVRs (blue) are shown separately. Deletions tend to be enriched for rare CNVRs, whereas duplications tend to be enriched in common variants

differentiation (Fst $=0.12-0.44), 7$ CNVRs had regulatory elements such as IncRNA and snoRNA within $\sim 300 \mathrm{~kb}$ from the CNVRs. Among the genic CNVRs, CNVR 380 $($ Fst $=0.21$; duplication), which is more frequent in JER $(\mathrm{MAF}=0.24)$ than in HOL $(\mathrm{MAF}=0.04)$, contains three genes, CLEC5A [42], TAR2R38 [43], and MGAM. The known functions of these genes include abnormal eating behaviour, bitter taste perception, and the synthesis of maltase glucoamylase, a starch digestive enzyme. Furthermore, CNVR 826, 1312, and 1458 overlap with genes that are known to regulate body size: LRRC49 [44], CA5A [45], and ADAMTS17 [46-48], respectively. Interestingly, these CNVRs are duplications and have a high allele frequency in JER $(\mathrm{MAF}=0.08-0.37)$, and a low allele frequency in HOL (MAF $=0-0.06)$.

Subsequently, we calculated Vst statistic, which is a widely used statistic in CNV studies [23, 49]. This statistic is analogous to Fst, but using LRR values instead of allele frequencies [28]. The Vst statistic ranges between 0 and 1 , where 1 indicates population differentiation. To strengthen our confidence in the high Fst outlier regions we compared Fst and Vst statistics. Firstly, we calculated Vst for 1464 CNVRs where Fst values are available. The Pearson correlation coefficient between Fst and Vst was low (0.22), and many selection candidate CNVRs that were found privately in Vst were either driven by rare CNVRs (less than 5 copies), or with a small number of SNPs (the numbers of average SNPs for top 20 Vst CNVRs and Fst CNVRs was 3.7 and 20.7 respectively;
Additional file 2: Figure S4 A-C). To correct for this, we removed CNVRs with less than $5 \mathrm{CNVs}$ are called from either HOL or JER population ( $n=1154$ CNVRs). We observed that this filtering removed outlier CNVRs that were private to Vst, that were consisting of a small number of SNPs. After this filter, the 32 high Fst CNVRs were kept and the correlation coefficient was $0.52(n=310$ CNVRs; Additional file 2: Figure S4 D-F). Also, CNVR 1458 which overlaps with ADAMTS17, showed a high Vst of 0.17 (mean Vst mean $=0.03$, Vst S.D. $=0.04)$. Furthermore, when the copy number filter was applied to both populations, and therefore both HOL and JER had more than five copies of CNVs at each CNVRs $(n=44)$, the correlation coefficient increased to 0.81 (Additional file 2: Figure S5).

\section{Linkage disequilibrium of CNVRs}

There has been a large number of genome-wide associations (GWAS) performed using SNPs in livestock species, aiming to unravel genomic regions related to phenotypes of interest [50]. This approach exploits a large number of tagging SNPs that are in sufficient LD with causal variants. Under this framework, genetic variation caused by the causal variants is captured by the tagging SNPs, without knowing the exact causal variants. Thus, the genome-wide level of LD between SNP markers and causal variants is an important foundation of GWAS [51]. We showed that CNVRs overlap with genes more often than would be expected by chance, and that CNVs are thus likely to have an influence on

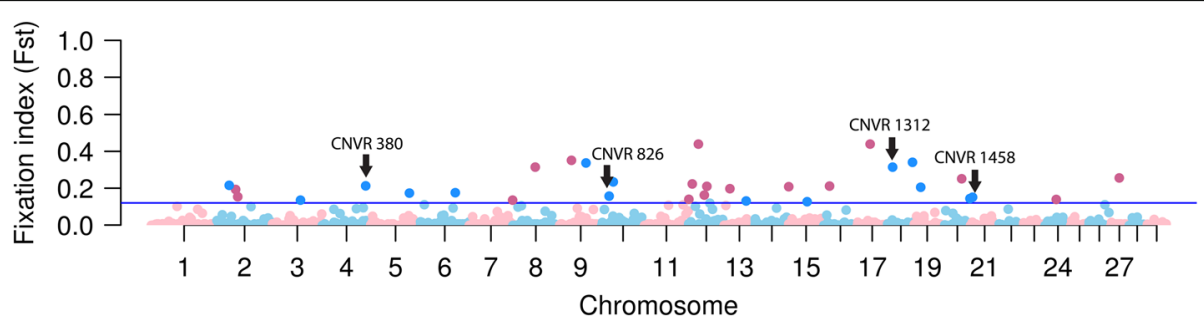

Fig. 4 Manhattan plot for population fixation index (Fst) of CNVRs between HOL and JER. Population fixation index (Fst) of bi-allelic CNVRs between HOL and JER is shown in a Manhattan plot. Seventeen intergenic CNVRs (magenta) and 15 genic CNVRs (dark blue) were above the suggestive threshold $(0.12$; Fst $>$ mean +3 S.D.). CNVRs containing candidate genes are marked with arrows 
phenotypes. The important follow-up question is whether the variations from $\mathrm{CNVs}$ are already captured by SNPs typed on commercial arrays, which are commonly used in livestock breeding programmes. We, therefore investigated pairwise LD between bi-allelic CNVRs and neighbouring SNPs on the BovineHD SNP chip. We observed generally low $r^{2}$, close to zero, regardless of the distance between CNVRs and SNPs (results not shown). Subsequently, we categorized CNVRs by their allele frequency and type to investigate whether these factors influence the degree of LD. Common CNVRs have markedly higher LD $\left(r^{2}=\sim 0.1\right.$ for deletion CNVRs at $\sim 10 \mathrm{~kb}$ distance), compared to other CNVR categories (Additional file 2: Figure S6). As common CNVRs had higher LD than the rest, we compared the LD of common CNVRs with the LD of SNPs in the same MAF range $(0.05 \leq \mathrm{MAF}<0.29$ for $\mathrm{HOL}$ and $0.05 \leq$ MAF $<0.37$ for JER). We observed distinctive difference in LD decay patterns between the CNVR-SNP pairs and SNP-SNP pairs (Fig. 5a and b). SNP-SNP LD follows a typical LD decay pattern where strong LD is observed with SNPs in vicinity and gradual decline as the distance increases, whereas CNVR-SNP LD does not follow this pattern. Also, compared to the CNVR-SNP LD $\left(r^{2}=\sim\right.$ 0.1 at $\sim 10 \mathrm{~kb}$ distance), the frequency matching SNPSNP LD was stronger $\left(r^{2}=\sim 0.5\right.$ at $\sim 10 \mathrm{~kb}$ distance). Afterwards, we used another metric, taggability, to assess LD. Taggability is the maximum $r^{2}$ among the $r^{2}$ values that are obtained from a variant of interest and SNP pairs. We calculated taggability for SNP-SNP pairs and CNVR-SNP pairs. For the CNVR-SNP pairs, we considered common deletion CNVRs only, as they showed the highest LD in the previous analyses. Then, mean taggability for each MAF class (bin size $=0.05$ ) was plotted (Fig. $5 \mathrm{c}$ and d). The mean taggability of common deletion CNVRs is low $(<0.1)$ when MAF is below 0.05 , and it increases as MAF increases. The SNP mean taggability follows the same pattern as shown in common deletion
CNVRs. However, in spite of the similar pattern, common deletion CNVRs taggability is below the level of the SNP taggability. This shows that there is a gap in SNP taggability and CNVR taggability.

\section{Interesting CNVR}

A large number of QTLs has been identified from various GWAS on a wide range of traits. As most GWAS have been done using SNP markers, chances are that genetic variation caused by $\mathrm{CNVs}$ could have been captured by QTLs that are in a high-to-perfect LD $\left(r^{2}=\sim 1\right)$ with the CNVs. Hence, inspecting CNVRs that are in high LD with QTLs is a preliminary step to identify potentially causal CNVs. To identify candidate causal CNVs, we subset the CNVR-QTL pairs, from the total CNVR-SNP pairs, based on the QTL information from the animal QTLdb [52]. We then subset the CNVR-QTL pairs further based on $r^{2}$, and kept high LD CNVR-QTL pairs only.

In total $\sim 100,000$ bovine QTLs for various traits have been reported in the animal QTL database, and we identified 2519 QTLs to be paired with 679 CNVRs within a distance of $100 \mathrm{~kb}$ in the HOL population. Among these, CNVR 547 (BTA6:84,395,081-84,428,819, deletion, MAF = 0.24 ) had the highest LD with 13 QTLs (average $r^{2}=0.59$; $\left.\max r^{2}=0.74\right)$. The 13 QTLs were associated with casein proteins, which constitute four out of six bovine milk proteins. The four genes coding for the casein proteins are located in the so called casein cluster, which is $\sim 1 \mathrm{Mb}$ distant region from CNVR 547 (BTA6:85.4-85.6 Mb). Given the degree of LD for CNVR 547 and the QTLs that is lower than perfect linkage, it is unlikely that the CNVR 547 is the causal variant for the casein protein traits. Nevertheless, CNVR 547 was an interesting variant as it was private to in HOL population with high MAF (0.24), and was close to the casein cluster that are highly relevant for dairy production.

Assuming that CNVR 547 is not the causal variant for the casein traits, a possible explanation for the high
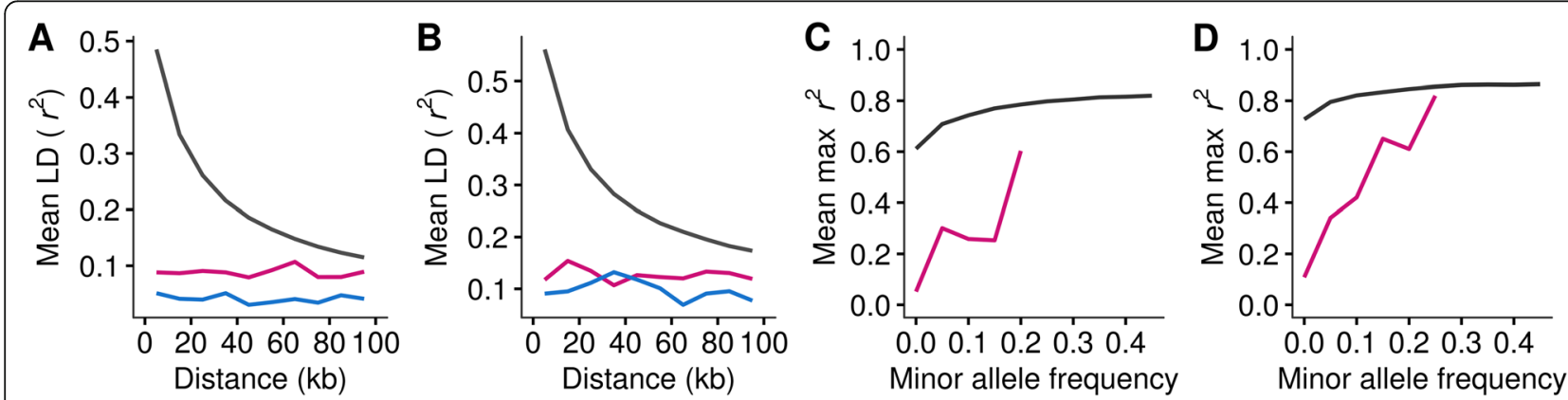

Fig. 5 Linkage disequilibrium properties of CNVRs. Average strength of linkage disequilibrium (mean $r^{2}$ ) as a function of distance from a SNP is shown for HOL (a) and JER (b). Common CNVRs ( $0.05 \leq$ MAF) were used for the calculation; common deletion CNVRs (magenta) and common duplication CNVRs (blue) are shown together with common SNPs (black) for comparison. Taggability for HOL (c) and JER (d) was expressed as ratio of variants in high LD $\left(r^{2}>0.8\right)$ with SNPs within $100 \mathrm{~kb}$ distance. Common deletion CNVRs (magenta) and common SNPs (black) are shown in the figure. Illumina BovineHD Genotyping BeadChip SNP set was used for the LD calculation 
MAF can be selective sweeps. Selective sweeps increase allele frequencies of neutral variants that are in LD with the selection target variant, which in this case probably is the casein cluster. Two studies of Holstein populations support this hypothesis. Firstly, one selective sweep study in a German Holstein population revealed an extended range of LD in haplotypes that contain the casein cluster [53]. Secondly, GWA study on casein traits in a Danish Holstein population identified a broad GWAS peak (BTA6:60-100 Mb) that contains the casein cluster [54]. The broad GWAS peak also indicate high LD in this regions, that matched with the findings from Qanbari et al. [53]

Another explanation for the high MAF of CNVR 547 might be the direct selection on the variant itself. For instance, CNVR 547 overlaps with the UGT2B4 gene, which is involved in the detoxification pathway of exogenous compounds [55]. To see whether CNVR 547 overlaps with regulatory elements, besides overlapping with the upstream region of the UGT2B4 gene directly, we called promoters and enhancers from ChipSeq data from Villar et al. [56]. CNVR 547 overlaps not only with the upstream (a start codon and the first two exons), but also with the enhancer of UGT2B4 (BTA6: 84,413,246$84,413,740)$, and is thus likely to disrupt the function of the UGT2B4 gene. To summarize, our analyses imply that a high MAF of CNVR 547 might be due the selective sweep in the casein cluster or the consequence of direct selection on CNVR 547 itself due to the functional impact of the overlap with UGT2B4 and its enhancer. Nonetheless, we cannot exclude drift as a possible driver for the high allele frequency of the CNVR 547.

\section{Discussion}

In this study, we discovered CNVs using bovine high density SNP array data. Using CNVRs that are constructed using the CNVs, we reported the functional impact and population genetic features of the CNVRs. They are further discussed below.

\section{CNV discovery in the genome build ARS-UCD1.2}

We observed different $\mathrm{CNV}$ discovery results between UMD3.1 and ARD-UCD1.2. The different results were to be expected, given the different sequencing platforms used for the assemblies. Long-read sequencing platforms are shown to perform better in retrieving repeat regions, which is considered to be challenging in short-read sequencing [57]. Among others, the most intriguing difference was observed for the BTA12:70-77 MB region. Based on the changes in BovineHD SNPs between UMD3.1 and ARS-UCD1.2, we postulated that the two genomes assemblies differ in this regions largely. Subsequently, the changes in the genome assemblies led to different $\mathrm{CNV}$ discovery results. We, then, further postulated that this region (BTA12:70-77 Mb in UMD 3.1) might contain repeated sequences, rather than the reported CNV, for two reasons. Firstly, the SNP density in this region is a quarter of the genome-wide average SNP density in UMD3.1 (71 SNPs/Mb and 292 SNPs/ $\mathrm{Mb}$, respectively; Additional file 2: Figure S2). SNP probes in repeat regions can reduce specificity of hybridization, and hence are often filtered out during SNP probe selection [58-60], which can explain why some regions show a sharp decrease in SNP density. Secondly, SNP probes in segmental duplications (sequence identity $>90 \%$ ) can induce confounded deletion calls due to cross-hybridization of paralogous sequences [61]. Our data set based on UMD3.1 was indeed enriched for a large number of deletion calls in this region. We regard this large difference as evidence underlining the importance of the quality of the reference genomes and the impact this has on $\mathrm{CNV}$ calling results.

\section{Functional impact of CNVRs}

In our functional impact analyses, we showed that the overlap between genes and CNVRs is higher than the overlap in a neutral scenario. This finding is in line with human and rat $\mathrm{CNV}$ studies, which showed that the overlap between $\mathrm{CNVs}$ and genes is significantly higher than expected by chance [62-64]. These studies were based on medium-to-large size human CNVs, and rat $\mathrm{CNVs}$ were found from exome arrays (CNV length ranged between 5 and $256 \mathrm{~kb}$ ). However, more recent studies, based on a finer resolution of CNVs, concluded that $\mathrm{CNVs}$ are biased away from genes and functional elements [5, 65-67].

Also, we observed that MAF have impact on different types of overlap between genes and CNVRs. In our findings, common deletion CNVRs were biased away from the genic regions, yet the common duplication CNVRs were skewed toward the genic part. This was contradicting with findings from another study, which showed both common deletions and duplications are skewed away from genic part [65].

We assume that these conflicting findings might arise from a curation of SNP array based CNVs in our dataset, which is affected by an ascertainment bias. An ascertainment bias of SNPs in commercial arrays can introduce a two-fold bias in CNV discovery. Firstly, the SNP density of a given array will constrain the size of CNVs that can be discovered. Secondly, SNP probes are designed such that complex regions, such as segmental duplications (SD), are under-represented [61, 68]. The SNP density of BovineHD BeadChip array in unique regions is 292 probes $/ \mathrm{Mb}$, whereas it drops to 95 probes/Mb in SD regions, showing a $67.5 \%$ reduction. Based on this, we speculate that the uneven genome-wide SNP coverage might introduce a systematic bias in CNV discovery. 
Taken together, the studies that focused on mid-sized CNVs [62-64] are in line with our findings, whereas studies based on tiling oligonucleotide microarrays [65] and whole genome sequencing data $[5,66]$, which can provide rather complete genome-wide coverage with a various size range of CNVs [4], show different results.

Furthermore, another layer of bias in CNV discovery using SNP array is that discovery of duplication is less sensitive than that of deletions. Consequently, most small CNVs are overwhelmingly deletions, whereas duplications usually are discovered based on relatively large number of SNPs than deletion, which makes duplications longer than deletions [4]. Indeed, in our CNV discovery, we found two folds more deletions than duplications (9171 vs. 4101$)$, and the mean length of duplication was longer $(44.2 \mathrm{~kb}$ vs $74.6 \mathrm{~kb})$. This deletionduplication bias might explain why common duplication CNVRs in our dataset are more likely to affect genic region compared to the rare duplication CNVRs, whereas it was the opposite in a study mentioned above [65].

The need to re-evaluate the functional impact of $\mathrm{CNVs}$, as $\mathrm{CNV}$ detection resolution became finer, along with the advancement in assay technologies and detection algorithms, was already pointed out [68]. Moreover, a recent study exploiting long-read sequencing data detected 237 and 34\% more insertions and deletions, respectively, compared to known variants detected from short-read sequencing data [69]. Taken together, the $\mathrm{CNVs}$ discovered in our dataset $(>1 \mathrm{~kb})$ were shown to be biased towards genic regions. However, we stress the need of re-visiting CNVs with finer resolution and unbiased genome-wide coverage, to fully comprehend their functional consequences in cattle genomes.

\section{Population genetics of CNVRs}

We explored the population genetics of CNVRs by examining the site frequency spectra and Fst. The frequency spectra differed for deletion CNVRs and duplication CNVRs. Given the skewed number of rare deletions and common duplications, we corroborate that deletions might be under stronger purifying selection. Nevertheless, as explained earlier, inherent bias in CNVs from SNP array (deletion discovery is more sensitive than duplication discovery), we cannot entirely exclude a possibility that the differed frequency spectra might be an artefact.

Furthermore, we used Fst to identify CNVRs that are highly diverged. Among the $32 \mathrm{CNVRs}$ that pass the threshold, of which 7 intergenic CNVRs had regulatory elements in neighbouring regions. This finding underlines that potential recent positive selection probably acted on regulatory elements. Among the 17 genic CNVRs, we identified CNVRs that overlap with interesting candidate genes. The CNVR 380 overlaps with
CLEC5A, TAR2R38 and MGAM gene that are related to taste perception and a digestion enzyme, maltase. One selective sweep study revealed that a region containing TAR2R38 and MGAM is highly diverged between dogs and wolves. Dogs produce a longer form of maltase than wolves, due to a $2 \mathrm{bp}$ deletion that disrupts the stop codon, and the same mutation was also seen in herbivore species (rabbits and cows) [70]. The longer form of maltase might be the consequence of adaptive evolution in response to a starch-rich diet during dog domestication. Given that the partial duplication of MGAM can lead to increased length of maltase, a high duplication frequency seen in the JER population (MAF $=0.24)$ might be a hint that feed related adaptive evolution occurred in the JER population. Also, we identified genes related to body size (LRRC49 in CNVR 826, CA5A in CNVR 1312, and ADAMTS17 in CNVR 1458). Among these genes, ADAMTS17 has been reported as one of the height determining genes in various species, such as cattle, horse, and human [46-48]. Also, a deletion variant overlapping with ADAMTS17 was shown to be highly diverged between HOL and JER in a previous study [66]. Given that CNVR 1452 we found is a duplication locus, it might be a different mutation than the one found by Mesbah-Uddin et al. [66]. Nonetheless, our and the previous findings revealed that CNVs overlapping with ADAMTS17 gene to be diverged between HOL and JER. This supports ADAMTS17 gene as a candidate gene that can explain the phenotypic differences (i.e. body size) between the two breeds.

Additionally, we used Vst analyses to confirm the selection candidate CNVRs based on Fst analyses. The preliminary results from Vst statistic from 1464 bialleleic CNVRs showed that extreme Vst could be obtained from very rare CNVs (less than $5 \mathrm{CNVs}$ observed) and short-sized variants. We consider correcting for these factors in analysing Vst statistic is crucial, as it could reduce falsely derived selection signal from false positive singletons [24]. We have seen that overall concordance between Vst and Fst was 0.52, when rare CNVRs (number of CNVs <5) were filtered out in either of the populations. Furthermore, when rare $\mathrm{CNVs}$ were filtered for both of the populations, which means CNVRs were present in both populations with more than 5 copies, the correlation coefficient was 0.81 . This number is slightly lower than 0.9, which was shown in human CNV study [49]. These findings underline high concordance of Fst and Vst when CNVRs are present in both populations with sufficient MAF. Thus, although we could obtain Vst confirmation for CNVR 1458, which overlaps with ADAMTA17, we could not obtain such confirmation for CNVRs that are at low MAF in either of the two populations. 


\section{Linkage disequilibrium of CNVRs}

To summarize our findings on LD properties of CNVRs, CNVRs are generally in low LD with SNPs, and CNVR taggability is lower than SNP taggability, which indicates a taggability gap. However, findings on the taggability are conflicting. Although some studies reported high CNV taggability [65, 68, 71-73], as high as SNP-SNP taggability, some studies reported low CNV taggability $[28,61,68,74,75]$ as shown in our results. The taggability gap can be explained by three factors. Firstly, LD is affected by allele frequency. High LD can be obtained when the allele frequencies of the two loci match [76]. Van Binsbergen et al. (2014) empirically showed that SNP-SNP pairs with small MAF difference $(<0.05)$ had high predicted LD $\left(r^{2}>0.8\right)$ using WGS data [77]. In our dataset, the majority of CNVRs is at low allele frequency (88 and $95 \%$ of CNVRs in JER and HOL are at MAF $\leq$ 0.05), whereas BovineHD SNPs are biased away from rare MAF $(10 \%$ of SNPs are at MAF $\leq 0.05)$. Thus, the allele frequencies of CNVRs and SNPs were largely unmatched, which can be explain the low LD. Secondly, deletions are tagged better than duplications. Even studies that found high taggability for common deletions, only found relatively poor taggability for duplications [5, 65,71 ]. This might be due to dispersal duplications [78], which relocate the duplicated segment of DNA in a different haplotype background than the "parental locus" [79]. Thus, the LD of duplications might be lower than that of deletions. Lastly, local SNP density can influence the level of LD. Redon et al. [28] and Locke et al. [75] suggested that a paucity of SNPs in repeat-rich regions to serve as potential tags can be an explanation for the taggability gap. Indeed, Cooper et al. [61] and McCarroll et al. [68] used different SNP sets in their CNV LD analyses. The first SNP set was HapMap phase 2 SNP set, which is known to cover the whole genome uniformly ( $3.1 \mathrm{M}$ probes). Next to this, they used SNP sets obtained from commercial SNP arrays, which have uneven SNP density along the genome (550 $\mathrm{K} \sim 1 \mathrm{M}$ probes). They found that $\sim 80 \%$ of CNVs are in high LD $\left(r^{2}>0.8\right)$ when HapMap phase 2 SNP set was used, whereas $50 \%$ of CNVs were in high LD with the commercial array SNP sets.

Based on our and previous findings, we postulate that LD between common deletion CNVRs and SNPs is not necessarily low. However, we could not obtain high LD with our CNVRs, because our CNVRs were skewed towards rare MAF. The MAF difference between CNVRs and SNPs can explain lower LD shown in rare CNVRs, compared to common CNVRs. However, as shown in another study [24], singletons found from PennCNV software could be false positives, which could lead to low LD as well. Thus, we could not exclude the possibility that the low LD in rare CNVRs was partly caused by false positive singleton $\mathrm{CNVs}$ driving low LD. Also, BovineHD SNPs were underrepresented in SD regions, where SNP probe design is difficult due to high sequence identity. Deprivation of SNPs in these regions probably led to lack of markers that can serve as tagging markers. Follow-up research using a SNP set that uniformly covers the whole bovine genome might unravel more complete LD properties of CNVs.

\section{Interesting CNVR}

Furthermore, in search of CNVRs that are causal variants of traits, we investigated CNVRs that are in high LD with known QTLs. CNVR 547 was shown to be in high LD with casein QTLs, although it was below perfect linkage, thus unlikely to be the causal variant. However, this opened up an interesting avenue to see the CNVR 547 in light of LD and selection. We proposed three possible explanation for CNVR 547 to reach high MAF:1) selective sweeps, 2) direct selection on CNVR 547 that affects the enhancer of UGT2B4 gene, and 3) drift. Although we could not unravel how CNVR 547 has reached high MAF in the current study, we deem it as an interesting case, which a CNVR can be understood in population genetics theories, such as selective sweeps and drift. Also, we had a limited number of CNVRs obtaining high LD with QTLs. This was partially due to because most CNVRs were rare, thus predisposed to have low LD. Therefore, re-visiting CNVR-QTL pairs, based on CNVs that are detected from a different platform (i.e. WGS) might reveal more candidate CNVs that might be the underlying causal variants of traits.

\section{Conclusions}

In this study, we discovered CNVs in bovine genomes and explored their functional impact and population genetics features. Using commercial high-density SNP arrays, we identified $14,272 \mathrm{CNVs}$, that built 1755 CNVRs (cover $\sim 2.8 \%$ of the bovine autosomes), and the CNVRs were further used as genetic loci this study. In the functional impact analyses, we showed that CNVRs are likely to have functional impact based on their overlap with genes. Also, we investigated CNVRs in light of population genetics. We identified 32 highly differentiated CNVRs between HOL and JER based on Fst values. Two of the highly diverged CNVRs overlapped with the ADAMTS17 gene and MGAM gene, which are involved in body size and starch digestion enzyme, respectively. In the LD analyses, CNVR-SNP LD was lower than SNP-SNP LD, mainly due to low MAF in CNVRs and uneven SNP density.

These findings together impose several implications for future CNV studies. The first implication is about the functional impact of CNVs. SNP based GWAS is a commonly used design to find functional SNPs that are 
associated with traits. Given the low CNVR-SNP LD, SNP based GWAS are unlikely to detect CNVRs with functional impact. Consequently, GWA studies that associate CNVRs and traits directly can add valuable insights into understanding economically important traits. Secondly, the low CNVR-SNP LD implies that the majority of CNVRs in our study is probably not captured in the current genomic prediction, where SNP markers are used. Thus, we underline the importance of follow-up studies on investigating methods to include CNVs in genomic prediction and evaluating the usefulness of CNVs in improving the accuracy of genomic prediction.

\section{Methods}

\section{Animal samples and ethics}

The study population consisted of two dairy cattle breeds, 331 Holstein Friesian (HOL), 115 Jersey (JER), as well as 29 crossbreds of HOL and JER. Among these, 18 HOL and 17 JER animals were cows and the rest were bulls. All samples were genotyped using an Illumina BovineHD Genotyping BeadChip (Illumina, San Diego, CA, USA), which contains 777,692 SNPs. All of these genotypes are owned by commercial dairy breeding company CRV (Arnhem, the Netherlands). The Genotype data was provided by CRV.

\section{Identification of CNVs}

We identified CNVs using PennCNV software [27] which exploits a Hidden Markov Model algorithm. For each individual, $\log \mathrm{R}$ ratio (LRR) and $\mathrm{B}$ allele frequency (BAF) per SNP were inferred using the Illumina Genome Studio software package (Illumina, San Diego, CA, USA). Autosomal SNPs of BovineHD Genotyping BeadChip ( $n=735,965$; Illumina, San Diego, CA, USA) were used, and their positions were based on the genome assembly ARS-UCD1.2. We called CNVs in 29 Bovine autosomes. The waviness in LRR values caused by GC contents were adjusted afterwards. We chose PennCNV software, together with BovineHD Genotyping BeadChip, as this method showed high confirmation based on qPCR validation in a previous study $(91.7 \%$ for CNVs found in multiple animals and $40 \%$ for singleton CNVs) [24]. After the initial CNV detection, poor quality individuals $(n=13)$ were filtered out with the default criteria suggested by the developer of the PennCNV software (LRR standard deviation $>0.30$, BAF standard deviation $>0.001$ and Waviness factor $>0.05$ ). Afterwards, the distribution of the number of $\mathrm{CNVs}$ per individual was inspected using QQ plots (Additional file 2: Figure S7). The distribution was continuous until 100, and individuals with more than $100 \mathrm{CNVs}$ largely deviated from the distribution $(n=10)$. The same filter on the distribution of the total length of CNVs per individual was applied and identified outlier samples $(n=11)$. These two filter steps identified 11 outlier individuals (among the 11 outlier animals identified in the second filter, 10 were identified as outliers in the first filter), and subsequently these individuals were removed to prevent the introduction of a large number of possible false positive CNVs. Lastly, we merged two adjacent CNVs that have the same copy number state, when the gap between the two CNVs was less than $10 \%$ of the total length, using the clean_cnv.pl script provided by PennCNV software, which resulted in 451 individuals with $14,272 \mathrm{CNVs}$ in the combined dataset of the two breeds.

\section{Constructing CNVRs}

The CNVs were aggregated into CNV regions (CNVR) based on $1 \mathrm{bp}$ overlap, following Redon et al. (2006) [28]. CNV regions that exclusively contain deletions or duplications were classified as deletion CNVRs and duplication CNVRs and treated as bi-allelic loci. In case of CNVRs that consisted of both deletions and duplications, we defined them as complex CNVRs. The CNVRs were compared together with SD and SNP density in. The SDs detected by Feng et al. [80] based on UMD3.1 were remapped to ARS-UCD1.2 using NCBI Genome Remapping Service. Afterwards, the density of SDs and SNPs were calculate for $5 \mathrm{Mb}$ bin using BEDtools [81]. Circos software [82] was used to visualize CNVRs, SD density, and SNP density. The length of CNVRs and SD was $\log$ transformed for the circular plot.

\section{Assessment of CNV discovery results}

We repeated the same CNV calling steps using 735,293 autosomal SNPs based on the genome assembly UMD3.1. After the initial $\mathrm{CNV}$ detection, the same quality control filters were applied as explained above. The default criteria filtered out 18 individuals, and another 11 outliers detected from QQ plots of the number of CNV per individual and the total length of $\mathrm{CNV}$ per individual were removed (Additional file 2: Figure S7). Subsequently, split $\mathrm{CNVs}$ that have small gaps in between were merged as described for ARS-UCD1.2. From the 447 individuals that passed the quality control criteria, 24,264 CNVs were called, and 1866 CNVRs were constructed as explained above. Finally, we compared the CNVs and CNVRs between the two different genome assemblies, UMD3.1 and ARS-UCD1.2, in terms of number and length.

\section{Functional impact of CNVRs}

The CNVRs were overlapped with gene annotations using Ensembl Variant Effect Predictor [83] (Cow release 95) to explore their functional impact. Subsequently, CNVRs were classified depending on their functional impact, as done in Conrad et al. [65]. First, we identified intergenic CNVRs, which did not overlap with genes, and genic CNVRs which overlapped with genes. Among 
the genic CNVRs, ones containing a complete gene or genes were classified as "whole gene". Genic CNVRs that overlapped with some part of genes were further classified as "intronic", when CNVRs overlapped with introns exclusively; as "stop codon", when CNVRs overlapped with stop codon; as "promoter region", when CNVRs included promoter region (500 bp from transcription start site). The remaining CNVRs that overlapped with an exon or exon(s) and intron(s) were considered as "exonic". In the case of CNVRs overlapping with more than one gene, and thus having more than one category assigned, (i.e. that contains a complete gene and also a promoter region of another gene), we assigned one unique category in the following order: 1) whole gene, 2) stop codon, 3) promoter region. With the steps explained above, each CNVR was assigned a unique category. Then, we investigated whether the functional impact classes were influenced by type of CNVRs (1125 deletion, 513 duplication, 117 complex CNVRs). Also, the influence of allele frequency on the functional impact classes were analysed and the allele frequency classes were defined as common (MAF $\geq 0.05$ in any population, 56 CNVRs), intermediate (0.1 $>$ MAF $\geq 0.01,267$ CNVRs), and rare (MAF $<0.01$ in HOL and JER, 115 CNVRs). To see whether the functional impact category differs significantly depending on type of CNVRs and MAF classes, Pearson's Chi-square tests were performed. Afterwards, CNVRs were classified depending and type and allele frequency in HOL and JER separately and the overlap with functional classes were analysed. Afterwards, we performed permutation tests to understand whether the observed overlap between CNVRs and a genomic feature is high or low, compared to random genomic regions. The permutation tests were performed with the $\mathrm{R}$ package "regioneR" [84]. We generated a random set of regions in the genome, with the same number and length of genomic features, and did this 1000 times. For each permutation, the number of overlaps between random CNVRs and the genomic features was recorded and then used to estimate the expected number of observations. The observed and the expected numbers of overlaps were then tested for significance (z-test). Subsequently, the PANTHER classification system [85] was used to perform gene ontology enrichment tests for the genes that overlapped with CNVRs. All known bovine genes (Ensembl release 95) were used as a reference set to test whether the CNVR overlapping genes were enriched for or deprived of a specific biological process, cellular composition, and molecular function, with False Discovery Rate correction $(\alpha<$ $0.05)$ for multiple tests.

\section{Population genetics of CNVRs}

We explored bi-allelic CNVRs in HOL and JER in light of population genetics. We genotyped bi-allelic CNVRs in HOL and JER into "+/+", "+/-", and "-/-", following McCarroll et al. [72]. These genotypes were used to calculate the allele frequency of each CNVR locus. Subsequently, we constructed site frequency spectra in $\mathrm{HOL}$ and JER to understand selection pressure on CNVRs. Wright's population differentiation index (Fst) [38] was used to investigate recent divergent selection in $\mathrm{HOL}$ and JER populations. Fst was calculated for 1471 biallelic CNVRs, using PLINK (version 1.9., http://pngu. mgh.harvard.edu/purcell/plink/) [86].

\section{Linkage disequilibrium of CNV}

We estimated the degree of LD between bi-allelic CNVRs and SNPs by calculating $r^{2}$ in the JER and HOL populations, respectively. To have a reference, we also estimated SNP-SNP LD, limited to SNPs with the same MAF range as common CNVRs $(0.05<\mathrm{MAF}<0.30$ for JER and $0.05<\mathrm{MAF}<0.24$ for HOL). The SNPs inside the CNVRs were masked to prevent a bias introduced during the phasing step, as done in Conrad et al. [65]. SNPs with low minor allele frequency (MAF $<0.001)$, with low call rates $(<90 \%)$, or with deviations from the Hardy-Weinberg equilibrium $\left(P<1 \mathrm{e}^{-9}\right)$ were removed. For CNVRs, the same filters were applied, except the call rate criteria. Phasing was done with Shapeit [87] and the $r^{2}$ values of CNVR-SNP pairs within a $100 \mathrm{~kb}$ distance were calculated in PLINK (version 1.9., http://pngu.mgh.harvard.edu/purcell/plink/) [86]. Afterward, QTLs that were shown to be significant in association studies were downloaded from Animal QTLdb [52] (release 37) and intersected with the CNVR-SNP pairs to see whether CNVRs are in high LD with known QTLs. To overlap the CNVR 547 and functional elements in bovine genomes, we used the data from Villar et al. [56]. We downloaded the ChipSeq data and aligned them to ARS-UCD1.2 using BWA-MEM (0.7.15) [88], and called the enhancers and promoters as explained in the original paper.

\section{Supplementary information}

Supplementary information accompanies this paper at https://doi.org/10. 1186/s12864-020-6496-1.

Additional file 1: Figure S1. Distribution of CNV length. Figure S2. Circular map of autosomal CNVRs in UMD3.1. Figure S3. Distribution of Fst values. Figure S4. Vst-Fst plots for 1464 biallelic CNVRs and after filtering for minimum of five copies of CNVs per CNVR in either of HOL and JER population. Figure S5. Vst-Fst plots after filtering for minimum of five copies of CNVs per CNVR in both HOL and JER populations. Figure S6. Linkage disequilibrium of CNVRs in different MAF classes. Figure S7. QQ plots for CNV quality control.

Additional file 2: Tables S1. Summary statistics for CNVs in ARS-UCD1.2 and UMD3.1. Table S2. Detailed features of CNVRs on autosomes identified in this study. Table S3. Chromosome-wide CNVR coverage based on ARSUCD1.2. Table S4. Chromosome-wide CNVR coverage based on UMD3.1. Table S5. Comparison between CNVRs identified in the present study with previous studies in terms of count and length. Table S6. CNVRs with high Fst values $(0.12<)$ and the genes affected by the high Fst CNVRs. 


\section{Abbreviations}

CNV: Copy number variation; CNVR: CNV region; Fst: Fixation index; HOL: Friesian Holstein; JER: Jersey; LD: Linkage disequilibrium; MAF: Minor allele frequency; QTL: Quantitative trait loci; SD: Segmental duplications; SNP: Single nucleotide polymorphism

\section{Acknowledgements}

The authors acknowledge CRV B.V. (Arnhem, the Netherlands) for providing the genotype data. The use of the HPC cluster has been made possible by CAT-AgroFood (Shared Research Facilities Wageningen UR).

\section{Authors' contributions}

All authors designed the study. YL performed the statistical analyses and drafted the manuscript under supervision of $A B$ and $M B$. $Y L, A B, M B, M G$, and $\mathrm{RV}$ interpreted the results. EM contributed to data collection, conception of the study, and manuscript revisions. All authors participated in discussions. All authors read and approved the final manuscript.

\section{Funding}

This study was financially supported by the Dutch Ministry of Economic Affairs (TKI Agri \& Food project 16022) and the Breed4Food partners Cobb Europe, CRV, Hendrix Genetics and Topigs Norsvin. MB was financially supported by NWO grant 016.Veni.181.050.

\section{Availability of data and materials}

The data that support the findings of this study are available from CRV B.V. (Arnhem, the Netherlands) but restrictions apply to the availability of these data, which were used under license for the current study, and so are not publicly available. Data are however available from the authors upon reasonable request and with permission of CRV B.V.

\section{Ethics approval and consent to participate}

The data used for this study were collected as part of routine data recording for a commercial breeding program. Samples collected for DNA extraction were only used for the breeding program. Data recording and sample collection were conducted strictly in line with Dutch law on the protection of animals (Gezondheids en welzijnswet voor dieren).

\section{Consent for publication}

Not applicable.

\section{Competing interests}

The authors declare that this study received funding from CRV B.V., Cobb, Hendrix Genetics, and TopigsNorsvin. All funders were involved in the study design, CRV B.V. performed data collection and was involved in preparation of the manuscript. MG is a member of the editorial board (Associate Editor) of BMC Genomics journal. All authors declare that the results are presented in full and as such present no conflict of interest.

\section{Author details}

${ }^{1}$ Wageningen University \& Research, Animal Breeding and Genomics, P.O. Box 338, Wageningen, AH 6700, the Netherlands. ${ }^{2}$ CRV, Arnhem, the Netherlands.

Received: 7 August 2019 Accepted: 14 January 2020 Published online: 28 January 2020

\section{References}

1. Feuk L, Carson AR, Scherer SW. Structural variation in the human genome. Nat Rev Genet. 2006;7(2):85-97.

2. lafrate AJ, Feuk L, Rivera MN, Listewnik ML, Donahoe PK, Qi Y, et al. Detection of large-scale variation in the human genome. Nat Genet. 2004;36(9):949-51 Available from: http://www.ncbi.nlm.nih.gov/ pubmed/15286789.

3. Sebat J, Lakshmi B, Troge J, Alexander J, Young J, Lundin P, et al. Largescale copy number polymorphism in the human genome. Science. 2004; 305(5683):525-8 Available from: http://www.ncbi.n/m.nih.gov/pubmed/152 73396.

4. Alkan C, Coe BP, Eichler EE. Genome structural variation discovery and genotyping. Nat Rev Genet. 2011;12(5):363-76. https://doi.org/10.1038/ nrg2958.
5. Sudmant PH, Rausch T, Gardner EJ, Handsaker RE, Abyzov A, Huddleston J, et al. An integrated map of structural variation in 2,504 human genomes. Nature. 2015;526(7571):75-81

6. Manolio TA, Collins FS, Cox NJ, Goldstein DB, Hindorff LA, Hunter DJ, et al. Finding the missing heritability of complex diseases. Nature. 2009;461(7265): 747-53. https://doi.org/10.1038/nature08494.

7. Eichler EE, Flint J, Gibson G, Kong A, Leal SM, Moore JH, et al. Missing heritability and strategies for finding the underlying causes of complex disease. Nat Rev Genet [Internet]. 2010;11(6):446-50. https://doi.org/10.1038/ nrg2809.

8. Bochukova EG, Huang N, Keogh J, Henning E, Purmann C, Blaszczyk K, et al. Large, rare chromosomal deletions associated with severe early-onset obesity. Nature. 2010;463(7281):666-70.

9. Coe BP, Witherspoon K, Rosenfeld JA, van Bon BWM, Vulto-van Silfhout AT, Bosco $P$, et al. Refining analyses of copy number variation identifies specific genes associated with developmental delay. Nat Genet. 2014;46(10):106371. https://doi.org/10.1038/ng.3092

10. Macé A, Tuke MA, Deelen P, Kristiansson K, Mattsson H, Nõukas M, et al. CNV-association meta-analysis in 191,161 European adults reveals new loci associated with anthropometric traits. Nat Commun. 2017;8(1):1-11.

11. Marshall CR, Howrigan DP, Merico D, Thiruvahindrapuram B, Wu W, Greer DS, et al. Contribution of copy number variants to schizophrenia from a genome-wide study of 41,321 subjects. Nat Genet. 2017;49(1):27-35.

12. Gonzalez E, Kulkarni H, Bolivar H, Mangano A, Sanchez R, Catano G, et al. The influence of CCL3L1 gene-containing segmental duplications on HIV-1/ AIDS susceptibility. Science. 2005;307(5714):1434-40.

13. Perry GH, Dominy NJ, Claw KG, Lee AS, Fiegler H, Redon R, et al. Diet and the evolution of human amylase gene copy number variation. Nat Genet. 2007:39(10):1256-60.

14. Rubin C-J, Megens H-J, Barrio AM, Maqbool K, Sayyab S, Schwochow D, et al. Strong signatures of selection in the domestic pig genome. Proc Natl Acad Sci. 2012:109(48):19529-36.

15. Giuffra E, Tornsten A, Marklund S, Bongcam-rudlo E, Chardon P, Kijas MHJ, et al. A large duplication associated with dominant white color in pigs originated by homologous recombination between LINE elements flanking KIT. Mamm Genome. 2002:13:569-77.

16. Durkin K, Coppieters W, Drögüller C, Ahariz N, Cambisano N, Druet T, et al. Serial translocation by means of circular intermediates underlies colour sidedness in cattle. Nature. 2012;482(7383):81-4.

17. Xu L, Cole JB, Bickhart DM, Hou Y, Song J, VanRaden PM, et al. Genome wide CNV analysis reveals additional variants associated with milk production traits in Holsteins. BMC Genomics. 2014;15(1):1-10.

18. Zhou Y, Connor EE, Wiggans GR, Lu Y, Tempelman RJ, Schroeder SG, et al. Genome-wide copy number variant analysis reveals variants associated with 10 diverse production traits in Holstein cattle; 2018. p. 1-9.

19. Prinsen RTMM, Rossoni A, Gredler B, Bieber A, Bagnato A, Strillacci MG. A genome wide association study between CNVs and quantitative traits in Brown Swiss cattle. Livest Sci. 2017;202(December 2016):7-12. https://doi. org/10.1016/j.livsci.2017.05.011.

20. Durán Aguilar M, Román Ponce SI, Ruiz López FJ, González Padilla E, Vásquez Peláez CG, Bagnato A, et al. Genome-wide association study for milk somatic cell score in Holstein cattle using copy number variation as markers. J Anim Breed Genet. 2017;134(1):49-59.

21. Ben Sassi N, González-Recio Ó, de Paz-del Río R, Rodríguez-Ramilo ST, Fernández Al. Associated effects of copy number variants on economically important traits in Spanish Holstein dairy cattle. J Dairy Sci. 2016;99(8):6371-80 Available from: http://linkinghub.elsevier.com/retrieve/pii/S0022030216302740.

22. Xu L, Hou Y, Bickhart DM, Zhou Y, Hay EHA, Song J, et al. Populationgenetic properties of differentiated copy number variations in cattle. Sci Rep. 2016;6:1-8. https://doi.org/10.1038/srep23161.

23. Bickhart DM, Xu L, Hutchison JL, Cole JB, Null DJ, Schroeder SG, et al. Diversity and population-genetic properties of copy number variations and multicopy genes in cattle. DNA Res. 2016;23(3):253-62

24. Upadhyay M, da Silva VH, Megens HJ, Visker MHPW, Ajmone-Marsan P, Bâlteanu VA, et al. Distribution and functionality of copy number variation across European cattle populations. Front Genet. 2017:8(AUG):1-12.

25. Pierce MD, Dzama K, Muchadeyi FC. Genetic diversity of seven cattle breeds inferred using copy number variations. Front Genet. 2018;9(163):1-14.

26. USDA ARS. Bovine reference genome ARS-UCD1.2 . 2018 [cited 2018 Mar 23]. Available from: https://www.ncbi.nlm.nih.gov/assembly/GCA 002263795.2 
27. Wang K, Li M, Hadley D, Liu R, Glessner J, Grant SFA, et al. PennCNV: an integrated hidden Markov model designed for high-resolution copy number variation detection in whole-genome SNP genotyping data. Genome Res. 2007;17(11):1665-74.

28. Redon R, Ishikawa S, Fitch KR, Feuk L, Perry GH, Andrews TD, et al. Global variation in copy number in the human genome. Nature. 2006;444(7118): 444-54.

29. Jiang L, Jiang J, Yang J, Liu X, Wang J, Wang H, et al. Genome-wide detection of copy number variations using high-density SNP genotyping platforms in Holsteins. BMC Genomics. 2013;14(1):1-10.

30. Sasaki S, Watanabe T, Nishimura S, Sugimoto Y. Genome-wide identification of copy number variation using high-density single-nucleotide polymorphism array in Japanese black cattle. BMC Genet. 2016;17(1):1-9.

31. Prinsen RTMM, Strillacci MG, Schiavini F, Santus E, Rossoni A, Maurer V, et al. A genome-wide scan of copy number variants using high-density SNPs in Brown Swiss dairy cattle. Livest Sci. 2016;191:153-60 Available from: http://linkinghub.elsevier.com/retrieve/pii/S1871141316301779.

32. Nandolo W, Utsunomiya YT, Mészáros G, Wurzinger M, Khayadzadeh N, Torrecilha RBP, et al. Misidentification of runs of homozygosity islands in cattle caused by interference with copy number variation or large intermarker distances. Genet Sel Evol. 2018;50(43):1-13.

33. Hou Y, Bickhart DM, Hvinden ML, Li C, Song J, Boichard DA, et al. Fine mapping of copy number variations on two cattle genome assemblies using high density SNP array. BMC Genomics. 2012;13:376.

34. Lupski JR, Stankiewicz P. Genomic disorders: molecular mechanisms for rearrangements and conveyed phenotypes. PLoS Genet. 2005;1(6):0627-33.

35. Conrad DF, Hurles ME. The population genetics of structural variation. Nat Genet. 2007:39:s30-6.

36. The Bovine Hapmap Consortium. Genome-wide Survery of SNP variation uncovers the genetic structure of cattle breeds. Science. 2009;324(April):528-32.

37. Welch JL. Famous individuals in the history of the Jersey and HolsteinFriesian breeds. lowa State Univ Vet. 1940;2(3):111-40.

38. Wright S. Genetical structure of populations. Nature. 1950;166(4215):247-9.

39. Jakobsson M, Scholz SW, Scheet P, Gibbs JR, Vanliere JM, Fung H, et al. Genotype, haplotype and copy-number variation in worldwide human populations. Nature. 2008;451(February):998-1003.

40. The International HapMap Consortium. A haplotype map of the human genome. Nature. 2005;437(October):1299-320.

41. Xue Y, Sun D, Daly A, Yang F, Zhou X, Zhao M, et al. Adaptive Evolution of UGT2B17 Copy-Number Variation; 2008. p. 337-46.

42. Wade TD, Gordon S, Medland S, Bulik CM, Heath AC, Montgomery GW, et al. Genetic variants associated with disordered eating. Int J Eat Disord. 2014;46(6):594-608.

43. Destito MCS, Souza MM, Cirillo CA, Ledda M, Zamboni A, Martin N, et al. GWAS of human bitter taste perception identifies new loci and reveals additional complexity of bitter taste genetics. Hum Mol Genet. 2014;23(1):259-67.

44. Dickinson ME, Flenniken AM, Ji X, Teboul L, Wong MD, White JK, et al. Highthroughput discovery of novel developmental phenotypes. Nature. 2016; 537(7621):508-14

45. Smith CL, Blake JA, Kadin JA, Richardson JE, Bult CJ. Mouse Genome Database (MGD)-2018 : knowledgebase for the laboratory mouse. Nucleic Acids Res. 2018;46(October 2017):836-42.

46. Bouwman AC, Daetwyler HD, Chamberlain AJ, Ponce $C H$, Sargolzaei M, Schenkel FS, et al. Meta-analysis of genome-wide association studies for cattle stature identifies common genes that regulate body size in mammals. Nat Genet. 2018;50(March):362-7.

47. Frischknecht M, Flury C, Leeb T, Rieder S, Neuditschko M. Selection signatures in Shetland ponies. Anim Genet. 2016;47:370-2.

48. Lango Allen H, Estrada K, Lettre G, Berndt SI, Weedon MN, Rivadeneira F, Willer CJ, Jackson AU, Vedantam S, Raychaudhuri S, Ferreira T, Wood AR, Weyant RJ, Segrè AV, Speliotes EK, Wheeler E, Soranzo N, Park JH, Yang J, Gudbjartsson D, Heard-Costa NL, Rand HJ. Hundreds of variants clustered in genomic loci and biological pathways affect human height. Nature. 2010; 467(7317):832-8

49. Strillacci MG, Gorla E, Cozzi MC, Vevey M, Genova F, Scienski K, et al. A copy number variant scan in the autochthonous Valdostana red pied cattle breed and comparison with specialized dairy populations. PLoS One. 2018;13(9):1-18.

50. Sharma A, Lee JS, Dang CG, Sudrajad P, Kim HC, Yeon SH, et al. Stories and challenges of genome wide association studies in livestock - a review. Asian-Australas J Anima Sci. 2015;28(10):1371-9.
51. Visscher PM, Wray NR, Zhang Q, Sklar P, McCarthy Ml, Brown MA, et al. 10 Years of GWAS Discovery : Biology, Function, and, Translation. Am J Hum Genet. 2017;101(1):5-22.

52. Hu Z-L, Park C, Reecy J. Developmental progress and current status of the animal QTLdb. Nucleic Acids Res. 2016;44(D1):827-33.

53. Qanbari S, Pimentel ECG, Tetens J, Thaller G, Lichtner P, Sharifi AR, et al. A genome-wide scan for signatures of recent selection in Holstein cattle. Anim Genet. 2010;41:377-89.

54. Buitenhuis B, Poulsen NA, Gebreyesus G, Larsen LB. Estimation of genetic parameters and detection of chromosomal regions affecting the major milk proteins and their post translational modifications in Danish Holstein and Danish Jersey cattle. BMC Genet. 2016;17(114):1-12.

55. Barre L, Fournel-gigleux S, Finel M, Netter P, Magdalou J, Ouzzine M. Substrate specificity of the human UDP-glucuronosyltransferase UGT2B4 and UGT2B7. FEBS J. 2007;274:1256-64.

56. Villar D, Berthelot C, Flicek P, Odom DT, Villar D, Berthelot C, et al. Enhancer evolution across 20 mammalian species. Cell. 2015;160(3):554-66.

57. Nakano K, Shiroma A, Shimoji M, Tamotsu H. Advantages of genome sequencing by long-read sequencer using SMRT technology in medical area. Hum Cell. 2017;30(3):149-61.

58. ArrayGen. Custom array design process [Internet]. [cited 2020 Jan 22]. Available from: https://www.arraygen.com/single-nucleotide-polymorphism.php.

59. Koltai $\mathrm{H}$, Weingarten-baror $\mathrm{C}$. Specificity of DNA microarray hybridization : characterization, effectors and approaches for data correction. Nucleic Acids Res. 2008;36(7):2395-405.

60. Lemoine S, Combes F, Le Crom S. An evaluation of custom microarray applications : the oligonucleotide design challenge. Nucleic Acids Res. 2009; 37(6):1726-39.

61. Cooper GM, Zerr T, Kidd JM, Eichler EE, Nickerson DA. Systematic assessment of copy number variant detection. Nat Genet. 2008;40(10):1199-203.

62. Nguyen D, Webber C, Ponting CP. Bias of selection on human copy-number variants. PLoS Genet. 2006;2(2):198-207.

63. Guryev V, Saar K, Adamovic T, Verheul M, Van Heesch SAAC, Cook S, et al. Distribution and functional impact of DNA copy number variation in the rat. Nat Genet. 2008:40(5):538-45.

64. Cooper GM, Nickerson DA, Eichler EE. Mutational and selective effects on copy-number variants in the human genome. Nat Genet. 2007;39(7S):S22-9.

65. Conrad DF, Pinto D, Redon R, Feuk L, Gokcumen O, Zhang Y, et al. Origins and functional impact of copy number variation in the human genome. Nature. 2010;464(7289):704-12.

66. Mesbah-Uddin M, Guldbrandtsen B, Iso-Touru T, Vilkki J, De Koning D-J, Boichard D, et al. Genome-wide mapping of large deletions and their population-genetic properties in dairy cattle. DNA Res. 2017;25(September 2017):49-59.

67. Boussaha M, Esquerré D, Barbieri J, Djari A, Pinton A, Letaief $R$, et al. Genome-Wide Study of Structural Variants in Bovine Holstein, Montbéliarde and Normande Dairy Breeds. PLoS One. 2015;10(8):1-21.

68. McCarroll SA, Kuruvilla FG, Korn JM, Cawley S, Nemesh J, Wysoker A, et al. Integrated detection and population-genetic analysis of SNPS and copy number variation. Nat Genet. 2008;40(10):1166-74.

69. Audano PA, Sulovari A, Graves-lindsay TA, Li YI, Wilson RK, Eichler EE, et al. Characterizing the major structural variant alleles of the human genome resource characterizing the major structural variant alleles of the human genome. Cell. 2019;176(3):663-75.

70. Axelsson E, Ratnakumar A, Arendt ML, Maqbool K, Webster MT, Perloski M, et al. The genomic signature of dog domestication reveals adaptation to a starch-rich diet. Nature. 2013;495(7441):360-4.

71. Mills RE, Walter K, Stewart C, Handsaker RE, Chen K, Alkan C, et al. Mapping copy number variation by population-scale genome sequencing. Nature. 2011;470(7332):59-65.

72. Mccarroll SA, Hadnott TN, Perry GH, Sabeti PC, Zody MC, Barrett JC, et al. Common deletion polymorphisms in the human genome. Nat Commun. 2006;38(1):86-92.

73. Hinds DA, Kloek AP, Jen M, Chen X, Frazer KA. Common deletions and SNPs are in linkage disequilibrium in the human genome. Nat Genet. 2006;38(1):82-5

74. Kato M, Kawaguchi T, Ishikawa S, Umeda T, Nakamichi R, Shapero MH, et al. Population-genetic nature of copy number variations in the human genome. Hum Mol Genet. 2010;19(5):761-73.

75. Locke DP, Sharp AJ, Mccarroll SA, Mcgrath SD, Newman TL, Cheng Z, et al. Linkage disequilibrium and heritability of copy-number polymorphisms 
within duplicated regions of the human genome. Am J Hum Genet. 2006; 79(August):275-90

76. Wray NR. Allele frequencies and the $r 2$ measure of linkage disequilibrium : impact on design and interpretation of association studies. Twin Res Hum Genet. 2005;8(2):87-94.

77. Van Binsbergen R, Bink MCAM, Calus MPL, Van Eeuwijk FA, Hayes BJ, Hulsegge I, et al. Accuracy of imputation to whole-genome sequence data in Holstein Friesian cattle. Genet Sel Evol. 2014;46(41):1-13.

78. Gondo Y, Gardner JM, Nakatsu Y, Durham-pierre D, Deveaut SA, Kuper C, et al. High-frequency genetic reversion mediated by a DNA duplication : the mouse pink-eyed unstable mutation. Proc Nati Acad Sci. 1993; 90(January):297-301.

79. Schrider DR, Hahn MW. Lower linkage disequilibrium at CNVs is due to both recurrent mutation and transposing duplications. Mol Biol Evol. 2010;27(1):103-11.

80. Feng X, Jiang J, Padhi A, Ning C, Fu J, Wang A, et al. Characterization of genome-wide segmental duplications reveals a common genomic feature of association with immunity among domestic animals. BMC Genomics. 2017;18(1):1-11.

81. Quinlan AR, Hall IM. BEDTools: a flexible suite of utilities for comparing genomic features. Bioinformatics. 2010;26(6):841-2.

82. Krzywinski M, Schein J, Birol I, Connors J, Gascoyne R, Horsman D, et al. Circos: An information aesthetic for comparative genomics. Genome Res. 2009;604:1639-45.

83. McLaren W, Gil L, Hunt SE, Riat HS, Ritchie GRS, Thormann A, et al. The Ensembl Variant Effect Predictor. bioRxiv. 2016;042374 Available from: http:// biorxiv.org/content/early/2016/03/04/042374.abstract.

84. Gel B, Diez-Villanueva A, Serra E, Buschbeck M, Peinado MA, Malinverni R. regioneR : an R/bioconductor package for the association analysis of genomic regions based on permutation tests. Bioinformatics. 2016;32(2): 289-91.

85. Thomas PD, Campbell MJ, Kejariwal A, Mi H, Karlak B, Daverman R, et al. PANTHER: a library of protein families and subfamilies indexed by function. Genome Res. 2003;13(9):2129-41. https://doi.org/10.1101/gr.772403.

86. Purcell S, Neale B, Todd-brown K, Thomas L, Ferreira MAR, Bender D, et al. PLINK : a tool set for whole-genome association and population-based linkage analyses. Am J Hum Genet. 2007;81(September):559-75.

87. Delaneau O, Marchini J, Zagury J. A linear complexity phasing method for thousands of genomes. Nat Methods. 2012;9(2):179-81.

88. Li H. Aligning sequence reads, clone sequences and assembly contigs with BWA-MEM. arXiv Prepr arXiv. 2013;00(00):3 Available from: http://arxiv.org/ abs/1303.3997.

\section{Publisher's Note}

Springer Nature remains neutral with regard to jurisdictional claims in published maps and institutional affiliations.

Ready to submit your research? Choose BMC and benefit from:

- fast, convenient online submission

- thorough peer review by experienced researchers in your field

- rapid publication on acceptance

- support for research data, including large and complex data types

- gold Open Access which fosters wider collaboration and increased citations

- maximum visibility for your research: over $100 \mathrm{M}$ website views per year

At $\mathrm{BMC}$, research is always in progress.

Learn more biomedcentral.com/submissions 